\title{
THE RESEARCH ON GRAIN RESERVE INTELLIGENT AUDIT METHOD AND IMPLEMENTATION IN THREE-DIMENSIONAL STORES
}

\author{
Ying Lin ${ }^{1,2^{*}}$, Xiaohui Jiang ${ }^{1}$ \\ ${ }^{1}$ School of Management, Chongqing Jiao Tong University, Chongqing, China, 400074 \\ ${ }^{2}$ School of Electronic Information Engineering, Tianjin University, Tianjin, China, 300072 \\ *Corresponding author, Address: No. 66 Xuefudadao, Chongqing Jiao Tong University, \\ Nanan district, Chongqing Municipality, P. R. China, 400074. Tel: 023-66876662. Email: \\ Linyingdyh@yahoo.com.cn
}

Abstract: In order to eliminate the drawbacks in grain reserve management, a grain reserve intelligent audit method was designed. It was achieved by using the edge detection technology towards image samples to determine the edge of each object in the photograph, including the grain, the wall and benchmarks, and then separately holding pattern recognition to ascertain the identity of each object. Then according to the basic theories of 3-D reconstruction technology, combining with the location information of these objects in the original photographs, the underlying quantitative information could be dug out. At last, using grain weight measuring algorithm, which had been combined with the perspective error correction method, achieve the real-time and precise audit to the grain in stock and each installment.

Keywords: Grain reserve; Intelligent audit; Image recognition; Perspective error

\section{INTRODUCTION}

Analyzing the drawbacks in grain reserve management of the China for the last years, such as virtual storage, false discount and so on, it reveals that the supervision and audit system are not effective, mainly because of the contradiction between the high supervisory requirements caused by the 
geographical dispersion of the granaries and the serious lack of powerful management technologies in reality. Currently, at home or abroad, the research on grain situation's surveillance (mainly including temperature, humidity and pest) have been conducted in depth, achieving several good results (Wei et al., 2004; Li et al., 2003). But on the area of grain audit, because an effective way to determine the weight of a large-scale object hasn't been designed out, this becomes the major bottleneck in grain reserve audit. Therefore, the key of eliminating the drawbacks is to use modern technologies to solve the problem of grain's weight determination in order to make the entire remote supervision comes true, instead of the manned field operations.

This paper mainly introduces an intelligent audit method in grain reserve management, based on the image recognition. As for the grain reserve audit, it includes the audit in two aspects, the weight and the time. So when designing the audit method, we focus on resolving the following three problems. The first is the recognition of a simple-frame image. Because, under the current technologies, it is relatively difficult to achieve dynamicflow recognition, a feasible idea is resorting to the recognition of numbers of simple-frame static images to refer the dynamic-flow recognition, in order to realize the real-time supervision and recognition. This is the foundation of this intelligent audit method. Further more, the installment judgment, as concerned to grain audit that is the judgment of the starting and ending between which each batch of grain were transport into or out of the stores. The last is perspective error correction. Because of the influence of video camera's view angle, the far object is compress to smaller than the near one. So there must be a certain perspective error in the data dogged from the plane image, which will have seriously influence on the accuracy of recognition (Haven \& Betty, 1977). So when designing the grain weight determine algorithm, the interference of the perspective error must be effectively excluded.

\section{GRAIN RESERVE INTELLIGENT AUDIT METHOD}

Generally, there are two approaches for grain weight measurement, the approach based on the direct measurement and the approach based on recognition. The former is usually to set up a dedicated channel and use correlative facilities to measure the weight, by which we can know the weight of each batch of the grain and the amount in stock. But this law requires a high cost in investment and maintenance. From the perspective of supervision, it asks for a high requirement of standardized operating, so if the grain custodians have illegal motivation, he also could carry out his 
illegal action easily. Therefore, using this approach to change the weak power of supervision is largely meaningless.

Currently, the approach based on the recognition, has rarely to be seen in reports (in the field of grain management). Image recognition, have two approaches which are worthy of paying closely attention to. The first is based on the distance measuring, which can be come true by using of the infrared or laser scanning to measure the distance between the probe and any point on the surface of an object. Through multi-detection, we can deduce the fitting three-dimensional shape of the object, from which we can calculate the bulk date of the digital object body. This approach asks for a long time and a high cost of equipments, so it is obviously not suitable for such a large amount of dynamic sites in grain reserve audit. Simultaneously, we can know that the key of this 3-D reconstruction, based on pattern recognition, is the third dimension's infers, by our experiential knowledge. (Qing et al., 2005).

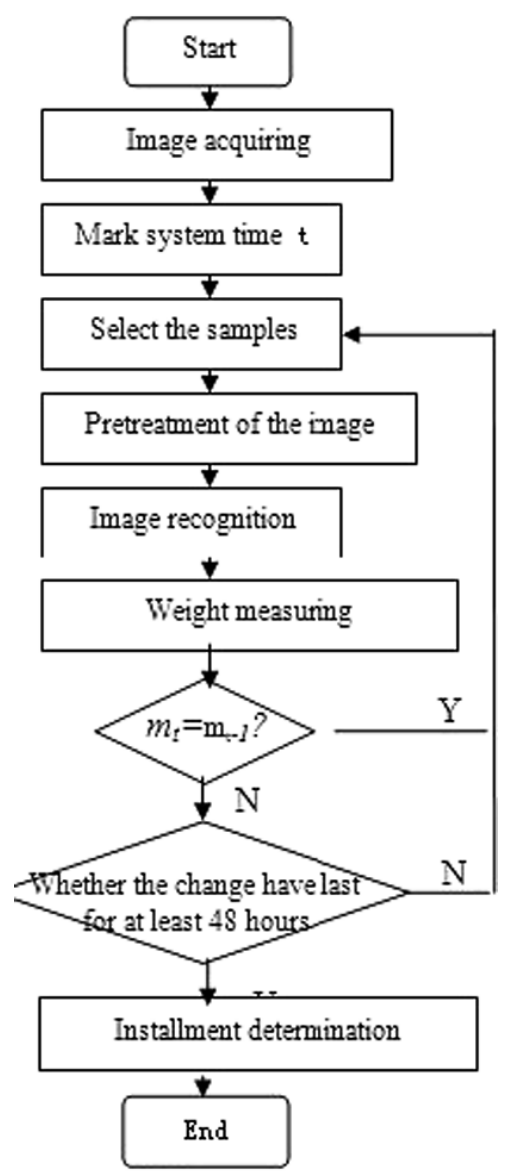

Fig. 1. Grain reserve intelligent audit method flowchart 
The other is based on plane image recognition, reaching the identification purpose by pattern recognition. Obviously it can also achieve the dynamicflow recognition by the contrast between consecutive simple-frames. And this method can achieve remote automatic control just under the supervisory camera in granary, thereby significantly reducing supervisory strength.

Based on the above analysis, we design a grain reserve audit method, whose audit processes are as follows: At first, aiming at the requirement of quantitative information in grain weight measuring algorithm, we should correspondingly do some pretreatment in granary site. Secondly, after the dynamic image flow, which was taken by video camera, was transmitted to the center computer through the Internet, the system will automatically select a simple-frame image as a sample by a fixed time interval. Then the sample will be taken to edge detection and image recognition, in order to dig out the underlying quantitative information. Further more we use the grain weight measuring algorithm, which have been combined with the perspective error correction method, achieving the determination of grain weight. Finally, according to the fixed time intervals of the consecutive samples, we can infer each installment from the grain weight and time (as shown in figure 1).

\section{IMPLEMENTATION}

The implementation of this audit method can be divided into four steps, pretreatment of grain storage site, mining the parameters of quantitative information, the grain weight measurement and the installment judgment. A experiment was held in the three-dimensional warehouse, a typical way of grain reserve.

\subsection{Pretreatment of grain storage site}

According to the characteristics of the three-dimensional warehouse, the pretreatment was carried out to satisfy the need of mining the underlying quantitative information in the following steps. It refers to, firstly, setting up the supervisory cameras in the appropriate positions. Secondly, painting two benchmarks on the inner wall of the granary, using a color which is quite different from the grain and the granary wall in color and grayscale features(in accordance with this standard to paint the benchmarks is to distinguish the benchmarks from other objects easily). The height of benchmark A is the same height with the camera, while benchmark B is in a certain distance below benchmark A (as shown in Figure 2).

In this step, the information which can be directly measured out includes: three-dimensional warehouse's bottom area $\mathrm{S}$, the camera and benchmark A's vertical height $\mathrm{H}$, the distance a between benchmark A and benchmark $\mathrm{B}$. 


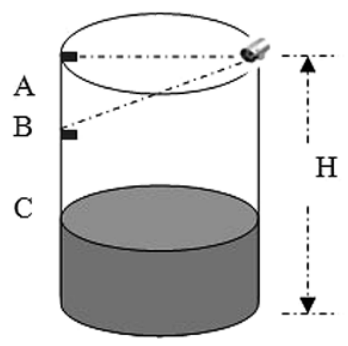

Fig. 2. Picture show of Three-dimensional stores

\subsection{Mining the parameters of quantitative information}

This step is the key of this intelligent audit method. Concretely, it can be achieve by the following two stages.

Firstly, select a simple-frame image sample from the dynamic flows by a a fixed time interval and evaluate it (the purpose is to exclude the unsatisfactory samples which have too much noise). Then with the computer's system time to mark the time for these simple-frame image samples, intending to be more facilitate to judge each batch's starting and ending of time. Evaluation of the selected samples is accomplished by scanning the image sample point-by-point, so as to form the phase-gray matrix. Then combining the basic principles of edge detection technology (Canny, 1986; Demigny, 2002), contrast the grayscale values of each point with the direction of the gradient near it, by which the image is divided into a set of meaningful regions (Liu, 2001) (each region can be considered to be a kind of object). Therefore, through the contrast of the regions with the number of the objects we have known in advance, can prove whether there is too much noise.

Secondly, to the eligible samples, we hold the image recognition with each meaningful region in the sample, in order to ascertain the identity of each object. Here, we use pattern recognition, which includes two major steps, the establishment of the standard database (that is, all of the information of possible objects and their parameters are established and saved in a database in advance), and the establishment of the test data (Cheng, 2006).

In response to the determination of grain weigh, the main task of recognition includes the identity of two benchmarks and their location in the original image, the identity of the grain surface and its location, from which we can also know the number of the pixel $\left(N_{l}\right)$ between benchmark A and B in the original image, and the number of the pixel $\left(N_{2}\right)$ between benchmark $A$ and grain surface $C$. Therefore, the information need to be saved in the standard database includes, the data of benchmark and grain surface's 
features, mainly including the color feature, the number-ration of pixel of the benchmark in vertical and horizontal directions, the grayscale feature and the texture information.

The determination of the identity of the benchmarks (or grain surface) is through comparing the test data with the standard data. For the accuracy close to $100 \%$, we make certain of the identity of the benchmarks (or grain surface) from the following four parameters: the color feature, the numberration of pixel of the benchmark in vertical and horizontal directions, the grayscale feature and the texture information.

If a test data matches with the standard data in over $95 \%$ confidence interval of the above four types of features, we can conclude that it is the benchmark (or grain surface). Based on this, the parameters $N_{l}$ and $N_{2}$ can be measured out easily.

\subsection{The grain weight measurement}

Till now, the known information includes the height of benchmark $A$ and $B$, the distance $a$ between benchmark $A$ and $B$, the height $H$ of the granary, the bottom area $S$ of the granary and density $\rho$. Then we can calculate out the weight of the grain in stock. The algorithm is as follows.

Step 1: according to the ratio of $N_{1} / N_{2}$ and the length of $a$, we can acquire the length of $A C$, namely $b=\left(N_{2} \times a\right) / N_{1}$, which is the length of $A C$ in the photograph reflect in the reality, having considered perspective err, and we can know that its length isn't unequal to $A C$.

Step 2: combining with the theory of image forming and plane geometry, we establish Cartesian coordinate system XY, as illustrated in figure 3. Then point $C^{\prime}$ is a point in a circle whose center is point $E$ and radius $r$ is the length between point $A$ and $E$, and we can know that $C, C^{\prime}, E$ has been in a same line; It comes to the equation:

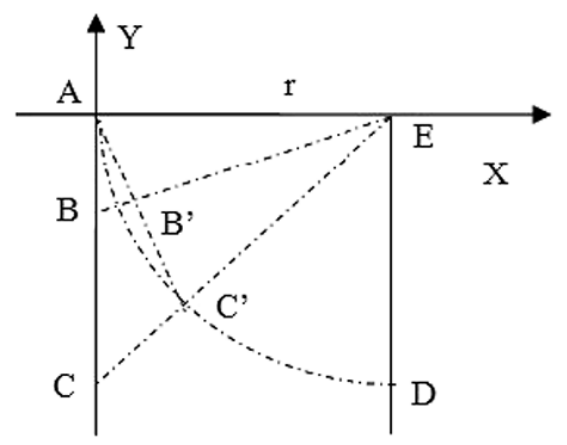

Fig. 3. Three-dimensional stores volume calculation model 


$$
(x-r)^{2}+y^{2}=r^{2}
$$

Step 3: according to the length of $b$, we can calculate out the coordinate of point $C^{\prime}(\mathrm{x}, \mathrm{y})$, and

$$
x^{2}+y^{2}=b^{2}
$$

From equation $\square$ and $\square$, we got:

$$
\left\{\begin{array}{l}
x=b^{2} / 2 r \\
y=\frac{-b \sqrt{4 r^{2}-b^{2}}}{2 r}
\end{array}\right.
$$

Step 4: as we have know the rdinate of point $E$, we can got the equation of line $C E$ from two point, point $E$ and $C^{\prime}$ :

$$
y=\frac{b \sqrt{4 r^{2}-b^{2}}}{2 r^{2}-b^{2}}(x-r)
$$

Then the coordinate of point $C$ can be calculated

$$
C=\left(0, \frac{b r \sqrt{4 r^{2}-b^{2}}}{2 r^{2}-b^{2}}\right)
$$

At last, we can obtain the height of the grain in the granary:

$$
h=H-\frac{b r \sqrt{4 r^{2}-b^{2}}}{2 r^{2}-b^{2}}
$$

So, the weight of the grain is easy to know by the equation of:

$$
m=\rho \times S \times h .
$$

Aggregate analysis, the calculating method of the height of the grain can resolve the perspective error problem of the objects in the image due to different shooting angles.

\subsection{The installment judgment}

The former steps are mainly to solve the problem of the grain weight determination. The grain reserve audit, as we have said before, contains two aspects, the weight and timing. Therefore, the installment judgment is also very necessary. The algorithm is as follows.

As shown in figure 2, according to the samples collected by every fixed time interval, after we have got the data of the grain's weight $m_{t}$ in the granary, then contrast the data with the former one $m_{t-1}$. If they are not equal, we think that the amount of grain is changing, this also indicates that this batch of grain haven't not yet complete, so we continue to consider the next 
sample. If $m_{t}=m_{t-1}$, then we make a judgment that whether the previous grain bulk hasn't been changed for over at lease 48 hours. If so, we can get the conclusion that this batch has completed, thus combining the initial time when grain weight began to change, the start time and end time of this batch can be inferred. And according to the difference between the two time, we can got the weight of this installment, namely $m=m_{t}-m_{o}$. If it is less than 48 hours, we will continue to select next sample and contrast their weight, until the grain weight hasn't changed for over 48 hours.

The accuracy of this intelligent audit method is mainly determined by the precision of the supervision cameras and image recognition, because of the relatively high stability of the images acquired from the close granary, the surrounding environment (such as brightness, humidity) generally will have little influence on it. And the quantity of the objects in the granary is relatively small, so the considering region and its edge of the images will be very clear, which is easier to achieve the edge detection and recognition. Currently, through experimental, the intelligent audit method is proved to be high accuracy, and with the continuous development of digital process technology, the accuracy of this grain reserve intelligent audit will also increasingly high.

\section{ACKNOWLEDGEMENTS}

This study has been funded by Finance Bureau of Chongqing Municipality. And sincerely thanks are also due to the Grain Administration of Chongqing Municipality for providing the experiment base for this study.

\section{REFERENCES}

Haven, Betty H. (1977). A Method for Minimizing Perspective Error. Journal of Physical Education and Recreation. 48(4), 74-77.

Wei Yong-lu, Zhang Ji-yue, Ji Wen-gang. (2005). Design of distributed control system for grain depot based on MODBUS/TCP Protocol. Beijing Institute of Petrochemical Technology Journal. Chn, 13(2): 2-4.

Li Jian-hua, Sun Hai-bo, Liu Zhan-liang, et al. (2003). The design and realization of supervising on provisions situation system [J]. Journal of the Hebei Academy of Sciences, 3(4): 224-227.

Qiang Wei-zhe, Song Guang-hua, Zheng Yao. (2005). 3D reconstruction based on image segmentation [J]. Computer Engineering and Application, 36: 77-82.

Liu li-bo. (2001). Summarization of segmental way of imagine. Ningxia Agricultural College Journal. Chn, 4(22), 51-53.

Canny, J. (1986). A Computational Approach to Edge Detection [J], IEEE Transations Pattern Analytical Machine Intelligent 8: 679-698.

D. Demigny. (2002). On Optimal Linear Filtering for Edge Detection, IEEE Trans. Image Processing, 2002, 9(11): 728-737.

Cheng Xiao-chun. (2006). A method of shape recognition [J]. Pattern Recognition and Artificial Intelligence, 6: 126-132. 